Post-print of: Economics Letters, 139(1), 2016, 72-75.

\title{
Affirmative Action through Minority Reserves: An Experimental Study on School Choice*
}

\author{
Flip Klijn ${ }^{\dagger} \quad$ Joana Pais ${ }^{\ddagger} \quad$ Marc Vorsatz ${ }^{\S}$
}

November 25, 2015

\begin{abstract}
Minority reserves are an affirmative action policy proposed by Hafalir et al. [8] in the context of school choice. In a laboratory experiment, we find that adding minority reserves to the GS and TTC mechanisms has positive effects on stability but is quite disappointing in terms of efficiency. Also GS induces higher rates of truth-telling by minority students and thus outclasses TTC.
\end{abstract}

Keywords: affirmative action; minority reserves; school choice; deferred acceptance; top trading cycles.

JEL-Numbers: C78, C91, C92, D78, I20.

${ }^{*}$ We are very grateful to the Department of Economics at Universidad Carlos III Madrid for providing laboratory facilities.

${ }^{\dagger}$ Corresponding author. Institute for Economic Analysis (CSIC) and Barcelona GSE, Campus UAB, 08193 Bellaterra (Barcelona), Spain; e-mail: flip.klijn@iae.csic.es. He gratefully acknowledges financial support from the Generalitat de Catalunya (2014-SGR-1064), the Spanish Ministry of Economy and Competitiveness through Plan Estatal de Investigación Científica y Técnica 2013-2016 (ECO2014-59302-P), and the Severo Ochoa Programme for Centres of Excellence in R\&D (SEV-2011-0075). He also gratefully acknowledges financial support from the Fundación Ramón Areces.

${ }^{\ddagger}$ ISEG, University of Lisbon and UECE-Research Unit on Complexity and Economics, Rua Miguel Lupi, 20, 1249-078, Lisboa, Portugal; e-mail: jpais@iseg.utl.pt. She gratefully acknowledges financial support from the Fundação para a Ciência e a Tecnologia under project reference no. PTDC/EGE-ECO/113403/2009.

$\S$ Departamento de Análisis Económico II, Universidad Nacional de Educación a Distancia (UNED), Paseo Senda del Rey 11, 28040 Madrid, Spain and Fundación de Estudios de Economía Aplicada (FEDEA), Calle Jorge Juan 46, 28001 Madrid, Spain; e-mail: mvorsatz@cee.uned.es. He gratefully acknowledges financial support from the Fundación Ramón Areces and the Spanish Ministry of Economy and Competitiveness (ECO2012-31985). 


\section{Introduction}

In the last decade, matching theory has been employed to accommodate affirmative action in school choice. For instance, Abdulkadiroğlu and Sönmez [2] and Abdulkadiroğlu [1] consider a maximum quota on the number of students from the same group a school can admit. However, Kojima [11] shows that for the widely studied Gale-Shapley (GS) and Top Trading Cycles mechanisms (TTC) maximum quotas can be detrimental to the very minorities they are supposed to help. To overcome this problematic feature, Hafalir et al. [8] propose to reserve seats for minority students. Loosely speaking, schools give higher priority to minority students up to the point where these students are interested in filling the reserved seats. Hafalir et al. [8] adapt GS and TTC to minority reserves and show that these mechanisms preserve the property of strategy-proofness (no student can ever benefit by misrepresenting her preferences) and that they are an improvement over majority quotas.

Still, the theoretical results may easily break down in practice. It is well documented in the experimental literature on school choice that agents do not always realize that it is in their best interest to reveal their true preferences when confronted with strategy-proof mechanisms. ${ }^{1}$ Since distinct strategy-proof mechanisms may be perceived differently, they could give rise to very different types of behavior and outcomes. Thus, it is the objective of our laboratory experiment to evaluate the effects of introducing minority reserves in GS and TTC.

\section{The experiment}

We consider the problem described in Table 1. Six students look for a seat at one of three schools. Four students $-M_{1}, M_{2}, M_{3}$, and $M_{4}$ - belong to the majority group, and two students $-m_{1}$ and $m_{2}$ - form the minority group. Each of the three schools $\left(s_{1}, s_{2}\right.$, and $\left.s_{3}\right)$ offers exactly two seats.

\begin{tabular}{|c|c|c|c|c|c|c|c|c|c|}
\hline & \multicolumn{6}{|c|}{ Preferences } & \multicolumn{3}{|c|}{ Priorities } \\
\hline & $M_{1}$ & $M_{2}$ & $M_{3}$ & $M_{4}$ & $m_{1}$ & $m_{2}$ & $s_{1}$ & $s_{2}$ & $s_{3}$ \\
\hline Best match & $s_{1}$ & $s_{1}$ & $s_{3}$ & $s_{3}$ & $s_{2}$ & $s_{2}$ & $m_{1}$ & $M_{3}$ & $M_{1}$ \\
\hline Second best match & $s_{2}$ & $s_{2}$ & $s_{1}$ & $s_{1}$ & $s_{3}$ & $s_{3}$ & $m_{2}$ & $M_{4}$ & $M_{2}$ \\
\hline Third best match & $s_{3}$ & $s_{3}$ & $s_{2}$ & $s_{2}$ & $s_{1}$ & $s_{1}$ & $M_{4}$ & $M_{2}$ & $m_{2}$ \\
\hline Fourth best match & & & & & & & $M_{3}$ & $M_{1}$ & $m_{1}$ \\
\hline Fifth best match & & & & & & & $M_{1}$ & $m_{1}$ & $M_{3}$ \\
\hline Sixth best match & & & & & & & $M_{2}$ & $m_{2}$ & $M_{4}$ \\
\hline
\end{tabular}

Table 1: Preferences of students over schools (left) and priority orderings of schools over students (right).

\footnotetext{
${ }^{1}$ See, for instance, Calsamiglia et al. [3], Chen and Kesten [4], Chen and Sönmez [5], Featherstone and Niederle [6], Guillen and Hing [7], Klijn et al. [10], and Pais and Pintér [12].
} 
We chose this problem for two reasons. First, in the absence of any positive discrimination, $m_{1}$ is in exactly the same situation as $M_{1}$ and $M_{3}$ (and $m_{2}$ faces exactly the same problem as $M_{2}$ and $M_{4}$ ). This symmetry helps us to evaluate the effect of minority reserves in a clear-cut way. Second, the unique Pareto-efficient outcome (assign each student to her top school) is obtained under all mechanisms only if submitted rankings satisfy stringent conditions. Essentially, subjects have to rank their best schools first. However, when subjects are not aware of strategy-proofness and look at their own priorities only, one cannot expect them to rank their best schools first, let alone to tell the truth. Yet, under minority reserves, $m_{1}$ can obtain a seat at her top school by ranking this school first independently of the behavior of the other subjects. This could reduce strategic uncertainty and induce all subjects to tell the truth more often.

Subjects assume the role of students, schools are not strategic players. Given the information in Table 1, the subjects' task is to submit a ranking over schools (not necessarily the true one) to be used by a central clearinghouse to assign students to schools. Subjects receive 12, 9, and $6 \mathrm{ECU}$ if they obtain a seat in their top, second most, and least preferred school, respectively. Our two control treatments are the standard GS student proposing deferred acceptance (GSs) and the standard TTC mechanism (TTCs). Following Hafalir et al. [8], the modified mechanisms, denoted by $G S m$ and TTCm, favor the minority group by obliging each school to reserve one seat to students from this group in case it is demanded.

Two matching mechanisms were played in each of the eight sessions. At the beginning of the session, subjects were anonymously matched into groups of six. Each subject received instructions for one of the four mechanisms and was told that she would play the game twice in different roles. Participants were also informed that after this first phase of two games they would take part in a second phase under a different matching mechanism. After completing the two games under the first mechanism, subjects received instructions for the second mechanism. The group assignment did not change and subjects took decisions in the same roles as before. Subjects were paid for their performance in one of the games (1 Euro per ECU earned). No feedback whatsoever was provided during the entire experiment. Subjects knew all procedures from the beginning. Sessions lasted about 75 minutes and subjects earned on average 12 Euro (including a 3 Euro show-up fee). In total, 175 subjects participated in the experiment.

\section{Results}

We analyze how the different mechanisms perform in terms of truth-telling. Afterwards, we study the implications of individual behavior on stability and welfare. 
Result 1. Minority reserves lead to more truth-telling only of $m_{1}$ under GS.

Evidence on Result 1. Table 2 shows the distributions of submitted rankings and the probabilies of truth-telling. For example, the notation $(2,3,1)$ indicates the ranking where a student lists her second most preferred school first, her least preferred school second, and her most preferred school last.

\begin{tabular}{|c|c|c|c|c|c|c|c|c|c|c|c|c|}
\hline \multirow[t]{2}{*}{ Mechanism } & \multicolumn{6}{|c|}{ Submitted ranking } & \multicolumn{6}{|c|}{ Truth-telling } \\
\hline & $(1,2,3)$ & $(1,3,2)$ & $(2,1,3)$ & $(2,3,1)$ & $(3,1,2)$ & $(3,2,1)$ & $\underline{M_{1}}$ & $M_{2}$ & $\underline{M_{3}}$ & $M_{4}$ & $\underline{m_{1}}$ & $m_{2}$ \\
\hline GSs & 0.39 & 0.02 & 0.34 & 0.11 & 0.02 & 0.12 & $\underline{0.47}$ & 0.37 & $\underline{0.37}$ & 0.27 & $\underline{0.44}$ & 0.37 \\
\hline GSm & 0.39 & 0.07 & 0.26 & 0.09 & 0.06 & 0.13 & $\underline{0.25}$ & 0.30 & $\underline{0.43}$ & 0.14 & $\underline{0.76}$ & 0.44 \\
\hline TTCs & 0.48 & 0.05 & 0.31 & 0.07 & 0.03 & 0.06 & $\underline{0.53}$ & 0.55 & $\underline{0.50}$ & 0.33 & $\underline{0.58}$ & 0.39 \\
\hline TTCm & 0.34 & 0.06 & 0.33 & 0.11 & 0.05 & 0.11 & $\underline{0.50}$ & 0.42 & $\underline{0.21}$ & 0.07 & $\underline{0.55}$ & 0.30 \\
\hline
\end{tabular}

Table 2: Distribution of submitted rankings (over all roles) and probability of truth-telling (by roles).

The left part of Table 2 reveals that many subjects do not realize that it is in their best interest to report preferences truthfully. Moreover, minority reserves do not increase the average truth-telling rate under GS and even lead to a drop under TTC. The effects of the different mechanisms and roles on truth-telling can be evaluated with the help of the right part of Table 2 . First, due to the symmetry of the game, one would expect that the proportion of truth-telling of the minority students is the same as that of their majority counterparts in the absence of minority reserves. Mann-Whitney U tests confirm this intuition in all cases. Moreover, as predicted, $m_{1}$ tells the truth more frequently in GSm than in GSs ( $p=0.0019$, two-sided). Also, she tells the truth more often than both $M_{1}$ ( $p=0.0015$, two-sided $)$ and $M_{3}$ ( $p=0.0107$, two-sided $)$ in GSm and more often than $M_{3}$ in TTCm ( $p=0.0368$, two-sided). On the other hand, minority reserves do not positively affect the level of truth-telling of this student under TTC. Finally, there is no evidence for spillover effects as no other student tells the truth significantly more often under minority reserves.

Result 2. Minority reserves increase stability.

Evidence on Result 2. A matching is stable in GSs and TTCs if for any student, all the schools she prefers to the one she is assigned to have exhausted their capacity with students that have higher priority. Therefore, in a stable matching no student can form a "blocking pair" with any school. Hafalir et al. [8] develop for GSm and TTCm an alternative notion of stability 
that gives less blocking power to majority students. ${ }^{2}$ The probabilities that a stable matching is reached are 0.3471 in GSs and 0.0460 in TTCs. Using the notion of Hafalir et al. [8], the corresponding numbers for GSm and TTCm are 0.3553 and 0.2403 , respectively. So, GS tends to be more stable than TTC and minority reserves increases stability for TTC while it almost does not affect GS. ${ }^{3}$ Also, with respect to the quality of the stable matchings reached we obtain that if one orders stable matchings according to average payoff, then the probability distribution over stable matchings delivering different average payoffs under GSm (TTCs) "almost" first-order stochastically dominates the distribution obtained under $G S s(T T C m){ }^{4}$

\begin{tabular}{lcccccccc}
\hline \hline \multirow{2}{*}{ Mechanism } & \multicolumn{7}{c}{ Students } \\
\cline { 2 - 9 } & $M_{1}$ & $M_{2}$ & $M_{3}$ & $M_{4}$ & $m_{1}$ & $m_{2}$ & Majority & Minority \\
\hline \hline GSs & 0.1938 & 0.1494 & 0.3878 & 0.0543 & 0.2301 & 0.1988 & 0.1963 & 0.2024 \\
GSm & 0.1972 & 0.1357 & 0.0941 & 0.0197 & 0.0960 & 0.0328 & 0.1117 & 0.0644 \\
TTCs & 0.3471 & 0.4674 & 0.3716 & 0.2721 & 0.2695 & 0.4913 & 0.3646 & 0.3804 \\
TTCm & 0.2813 & 0.3419 & 0.1931 & 0.0507 & 0.2162 & 0.0852 & 0.2168 & 0.1507 \\
\hline \hline
\end{tabular}

Table 3: Average probability of belonging to a blocking pair (standard definition for GSs and TTCs and notion of Hafalir et al. [8] for GSm and TTCm).

To complete the analysis on stability, we look into the expected probability of belonging to a blocking pair. Table 3 reveals, as expected, that in the standard mechanisms the average majority and the average minority student have roughly the same probability of being part of a blocking pair. This symmetry breaks down once minority reserves are implemented as the average probability with which a minority student belongs to a blocking pair is significantly reduced, while that of majority student is reduced to a lesser extent.

Result 3. Minority reserves slightly increase (decrease) expected payoffs under GS (TTC). They benefit minority students, but harm majority students.

Evidence on Result 3. Any successful discriminatory policy should increase the payoffs (or at least the average payoff) of minority students when minority reserves are introduced.

\footnotetext{
${ }^{2}$ We are grateful to an anonymous referee for the suggestion to compare the standard notion of stability for treatments GSs and TTCs with the modified notion of stability for treatments GSm and TTCm.

${ }^{3}$ Due to the very large number of recombinations, all pairwise comparisons are significant at $p=0.01$. This is also true for the remaining analysis on stability and our final result on payoffs.

${ }^{4}$ In each comparison there is one instance in which a probability mass of less than 0.002 goes in the wrong direction.
} 


\begin{tabular}{lcccccccc}
\hline \hline \multirow{2}{*}{ Mechanism } & \multirow{2}{*}{ Overall } & \multicolumn{7}{c}{ Students } \\
\cline { 3 - 9 } & & $M_{1}$ & $M_{2}$ & $M_{3}$ & $M_{4}$ & $m_{1}$ & $m_{2}$ & Minority \\
\hline \hline \multirow{2}{*}{ GSs } & 12.06 & 12.33 & 12.02 & 11.98 & 12.07 & 12.06 & 11.92 & 11.99 \\
& $(3.38)$ & $(4.59)$ & $(1.99)$ & $(5.55)$ & $(1.58)$ & $(4.25)$ & $(2.20)$ & $(6.65)$ \\
& & & & & & & & \\
GSm & 12.27 & 11.97 & 11.67 & 12.27 & 11.26 & 14.34 & 12.12 & 13.23 \\
& $(5.05)$ & $(8.09)$ & $(5.05)$ & $(3.93)$ & $(2.94)$ & $(3.19)$ & $(1.31)$ & $(3.20)$ \\
& & & & & & & & \\
$T T C s$ & 12.70 & 13.44 & 12.24 & 13.10 & 12.01 & 13.52 & 11.90 & 12.70 \\
& $(5.64)$ & $(4.34)$ & $(6.11)$ & $(5.19)$ & $(6.13)$ & $(4.13)$ & $(5.23)$ & $(9.61)$ \\
& & & & & & & & \\
& 12.25 & 12.29 & 11.33 & 12.16 & 11.80 & 13.44 & 12.47 & 12.96 \\
& $(5.26)$ & $(6.88)$ & $(5.18)$ & $(5.80)$ & $(2.48)$ & $(5.69)$ & $(2.99)$ & $(8.27)$ \\
\hline \hline
\end{tabular}

Table 4: Expected payoffs. In parentheses, we present variances.

Table 4 indicates that, on average, the introduction of minority reserves leads to slightly higher (lower) payoffs under GS (TTC). A closer inspection of the above table reveals that all majority students (except $M_{3}$ when we compare $G S m$ with $G S s$ ) obtain lower expected payoffs once minority reserves are introduced. On the contrary, minority students (except $m_{1}$ when we compare TTCm with TTCs) benefit from the introduction of minority reserves. In fact, if we consider minority students as a group, the expected rewards increase and their variance is reduced when minority reserves are introduced. Finally, a comparison of the full distributions of individual payoffs reveals that minority student $m_{1}$ 's payoffs under $G S m$ has first-order stochastic dominance over his payoff distribution under GSs and that the opposite holds for majority student $M_{4}$. On the other hand, for $M_{1}, M_{2}$, and $M_{3}$ the payoff distribution under TTCs dominates the distribution under TTCm.

\section{Conclusion}

We have analyzed experimentally the effects of minority reserves on the GS and the TTC mechanisms in a school choice problem. In contrast with Kawagoe et al. [9], our main finding highlights that adding minority reserves increases truth-telling only by some minority student in GS. The intuition that the GS mechanism performs better than the TTC mechanism in the considered problem is confirmed when one looks at stability and welfare. Finally, one should also keep in mind that our results were obtained in a simple setting with the flavor of a coordination game that puts pressure on the level of truth-telling but where minimum reserves could actually bring a clear improvement for all students in terms of efficiency, which we do not observe. 


\section{References}

[1] A Abdulkadiroğlu (2005). College admissions with affirmative action. International Journal of Game Theory 33(4): 535-549.

[2] A Abdulkadiroğlu and T Sönmez (2003). School choice: A mechanism design approach. American Economic Review 93(3): 729-747.

[3] C Calsamiglia, G Haeringer, and F Klijn (2010). Constrained school choice: An experimental study. American Economic Review 100(4): 1860-1874.

[4] Y Chen and O Kesten (2013). From Boston to Chinese parallel to deferred acceptance: Theory and experiments on a family of school choice mechanisms. Discussion Paper, Social Science Research Center Berlin (WZB).

[5] Y Chen and T Sönmez (2006). School choice: An experimental study. Journal of Economic Theory 127(1): 202-231.

[6] C Featherstone and M Niederle (2013). Improving on strategy-proof school choice mechanisms: An experimental investigation. Working Paper, Stanford University.

[7] P Guillen and A Hing (2014). Lying through their teeth: Third party advice and truth telling in a strategy proof mechanism. European Economic Review 70(1): 178-185.

[8] I Hafalir, M Yenmez, and M Yildirim (2013). Effective affirmative action in school choice. Theoretical Economics 8(2): 325-362.

[9] T Kawagoe, T Matsubae, and H Takizawa (2013). Affirmative action in school choice problem: An experiment. Working paper, Future University Hakodate, Hokkaido.

[10] F Klijn, J Pais, and M Vorsatz (2013). Preference intensities and risk aversion in school choice: A laboratory experiment. Experimental Economics 16(1): 1-22.

[11] F Kojima (2012). School choice: Impossibilities for affirmative action. Games and Economic Behavior 75(2): 685-693.

[12] J Pais and Á Pintér (2008). School choice and information: An experimental study on matching mechanisms. Games and Economic Behavior 64(1): 303-328. 